\title{
Systemic signalling in gene silencing
} $G$ ene silencing in plants is a genetic conresistance ${ }^{1,2}$, genome maintenance ${ }^{3}$ and developmental control ${ }^{4}$. We describe here our recent discovery that there is a systemic signal that can mediate gene silencing. From the gene-specificity of the systemic silencing, we infer that the signal molecule is likely to be a nucleic acid.

We studied Nicotiana benthamiana plants carrying a jellyfish green fluorescent protein (GFP) transgene ${ }^{5}$. We infiltrated leaves with strains of Agrobacterium tumefaciens carrying a GFP reporter gene. The bacterium contains a tumour-inducing (Ti) plasmid which can be modified in the transferred DNA (T-DNA) region to carry foreign genes into plant nuclear DNA.

Two days after infiltration, there was strong green fluorescence in the infiltrated region, indicating transfer of the T-DNA from the bacterium into the plant cell and expression of the GFP reporter gene (Fig. 1a). This strong fluorescence was superimposed over a weaker background fluorescence from the GFP found naturally in the transgenic plants. However, at the edge of the infiltrated zone there was a thin line of tissue in which the GFP fluorescence was absent, indicating that expression of the GFP transgene had been silenced (Fig. 1a).

Although the zone of GFP silencing did not spread further within the infiltrated leaf, by 18 days after infiltration there was silencing of the GFP transgene in the upper leaves of the infiltrated plant (Fig. 1b-d). In leaves of the axillary shoots (Fig. 1b) and in some of the uppermost leaves (Fig. 1d) there was complete silencing of the GFP transgene. The GFP transgene was not silenced if the Ti plasmid did not have a GFP coding sequence; if the cultures were prepared in the absence of acetosyringone as an inducer of DNA transfer into plant cells $^{6}$; or if the leaves were infiltrated with water (Fig. 1d). We conclude that the systemic silencing of the GFP transgene is a sequence-specific effect, following transient expression of the GFP reporter gene from the bacterium in the transgenic plants.

In subsequent tests, we found that systemic silencing of the GFP transgene was associated with reduced levels of GFP messenger RNA. We also found that an RNA virus vector, potato virus $\mathrm{X}$ carrying the GFP gene (PVX-GFP) ${ }^{7}$, failed to accumulate when inoculated into the GFP-silenced leaves. This resistance to PVX-GFP was sequence-specific because PVX with an insert of the GUS reporter gene accumulated to a high level. It is likely that this sequence-specific virus resistance and the systemic silencing are the result of the same mechanism targeted against the GFP

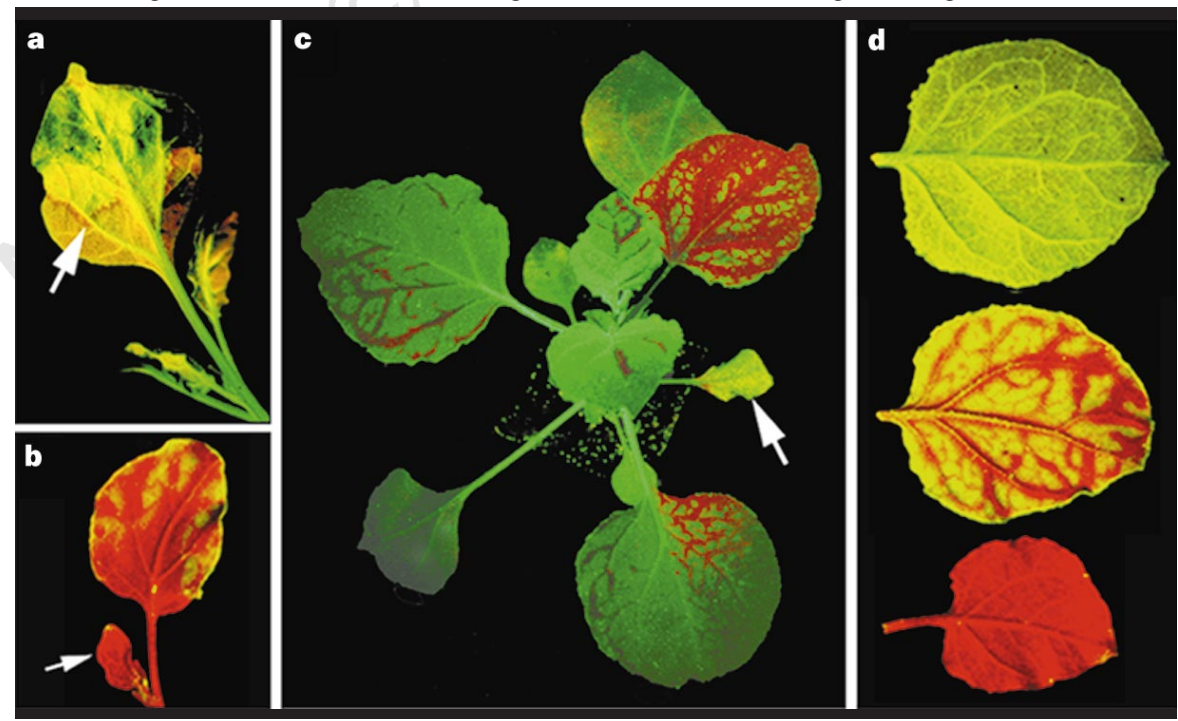

Figure 1 Expression of GFP in transgenic N. benthamiana. Under ultraviolet illumination, GFP appears green or yellow. In the absence of GFP, the tissue appears red owing to the fluorescence of chlorophyll. a, The leaf of GFP transgenic $N$. benthamiana infiltrated 2 days previously with $A$. tumefaciens carrying a GFP reporter gene. Arrow indicates the GFP-silenced boundary between the distal infiltrated zone (strong green fluorescence) and the proximal zone (weak green fluorescence due to the GFP transgene). b, Leaf and axillary shoot (arrow) of the upper part of a plant infiltrated 18 days previously, showing residual GFP in diffuse regions of the leaf and the extreme distal region of the axillary shoot. c, Intact GFP transgenic plant infiltrated 18 days previously in a lower leaf (arrow) showing the progression of GFP-silencing. d, Upper leaves emerging from the main stem of GFP plants infiltrated 18 days previously with water (top), or with the GFP strain of $A$. tumefaciens (middle and bottom). coding sequence of the viral and transgene RNAs.

Using several approaches, we failed to detect either A. tumefaciens cells or T-DNA in the systemic tissue, indicating that the silencing was caused by a signal molecule that moves out of the infiltrated leaf. The signal was produced when the GFP reporter gene was transferred to the cells of the GFP transgenic plant and is targeted against GFP RNA, indicating that the GFP, or the corresponding DNA or RNA, might be a component of the signal.

Of these, GFP is the least plausible candidate, because there is no mechanism known to us that explains how it could move systemically and specifically target the GFP RNAs. However, a nucleic acidbased signal could mediate sequencespecific gene silencing by forming a base-paired or triple helical structure with the target RNA. Moreover, a nucleic acid could move in the plant using the channels involved in virus or viroid movement.

Previous analyses have described nonclonal mosaics of gene silencing ${ }^{8,9}$ and inferred that an extracellular signal is involved, but these studies did not suggest that the signal would have the potential to move systemically. The systemic signal may be related to recent findings that gene silencing is associated with induced, natural defence against viruses ${ }^{1,2}$. The signal could move in the plant ahead of the inducing virus, so that antiviral gene silencing could delay spread of the infection front.

We are currently attempting to characterize the systemic signal of GFP gene silencing. Meanwhile, the system described here may provide an experimental system for testing the function of cloned plant genes and for dissecting the mechanism of gene silencing.

\section{Olivier Voinnet}

\section{David C. Baulcombe}

The Sainsbury Laboratory,

John Innes Centre, Colney,

Norwich NR4 7UH, UK

e-mail:baulcombe@bbsrc.ac.uk

1. Covey, S. N., Al-Kaff, N. S., Langara, A. \& Turner, D. S. Nature 385, 781-782 (1997).

2. Ratcliff, F., Harrison, B. D. \& Baulcombe, D. C. Science 276, 1558-1560 (1997).

Assaad, F. F., Tucker, K. L. \& Signer, E. R. Plant Mol. Biol. 22 1067-1085 (1993).

4. Boerjan, W., Bauw, G., van Montagu, M. \& Inzé, D. Plant Cell 6, 1401-1414 (1994).

5. Haseloff, J., Siemering, K. R., Prasher, D. C. \& Hodge, S. Proc. Natl Acad. Sci. USA 94, 2122-2127 (1997).

6. Ream, W. Annu. Rev. Phytopathol. 27, 583-618 (1989).

7. Baulcombe, D. C., Chapman, S. N. \& Santa Cruz, S. Plant J. 7, 1045-1053 (1995).

8. Jorgensen, R. A. Science 268, 686-691 (1995).

9. Kunz, C., Hanspeter, S., Stam, M., Kooter, J. M. \& Meins, F. J. Plant J. 10, 437-450 (1996). 